\title{
Observational Study on Myopia Progression in Children.
}

\author{
Dr. Harish Singh ${ }^{1}$, Xiao Shou Hua ${ }^{2}$, Rukhsar Ahmad ${ }^{3}$, Pankaj Kumar ${ }^{3}$, \\ Sui Jian ${ }^{4}$ \\ ${ }^{I}$ Department of ophthalmology, ${ }^{2}$ Department of clinical medicine, ${ }^{3}$ Department of general surgery, Medical \\ College of Jiangsu university, Zhenjian, 212001 China . \\ Corresponding author :Sui Jian,e-mail-jwcsj@163.com
}

\begin{abstract}
This review examin the trend toward greater rates of myopia as well as possible reasons for this increase rate in children. A lots of basic factors are responsible for myopia progress in children. An attempt to control childhood myopia progression have included various type of spectacles correction and contact lence use. Historical evidence indicates that corneal reshaping contact lenses may slow myopia progression in children. Like these there are various other factors which are responsible for myopia progression, like genetic defect, axial length, food habit, sex, race, hobbies.
\end{abstract}

Keywords: Children, corneal reshaping, genetic, axial length.

\section{Introduction}

Myopia in Greek words 'muopia' commonly known as nearsighted or short sighted. It's a condition of the eye where the light rays which should come directly on retina is not directly focusing on the retina but in front of retina .Most professional commonly correct myopia through the use of corrective lens such as glasses or contact lens.

Myopia is an important public health problem in asian countries like China Japan ,Singapore, where myopia rates are increasing over the past few decades .This condition have become a benign ocular disorder and also associated with potentially blinding condition such as pigmentery degeneration,retinal detachment ,premature cataract , and glaucoma, macular choroidal degeneration [1,21].The economic cost of correction of myopia with spectacles and contact lenses as the cost of optometry visit amount to billion of dollar per year.In general, high myopia is categorized as myopia of -6.00 or more diopter. And low myopia has a refractive range of -0.25 to $-3.00 \mathrm{D}$, and intermediate myopia is from -3.00 to $-5.00 \mathrm{D}$ and frequently manifest a scleral crescent formation. Congenital high myopia leads to increase in axial length and can be non progressive .It can also lead to retinopathy of prematurity abnormal ocular refractive component \{keratoconus, lenticonus, and spherophakia\}.

Systemic conditions, syndrome prematurity and associated low birth weight are major contributors to increased incidences of high myopia and myopia in general. Pathologic myopia is high myopia, with the presentation of progressive myopic changes in childhood that include increased axial length and fundus. The genetic basis of myopia has been well documented .The mode of transmission can be either autosomal dominant or recessive .However, environmental and cultural aspects also influence the development of myopia. The prevalence, progression, and age at onset of high myopia varies with ethnicity and gender. The rate of myopia is increased more in Chinese school children as compare to Japan, where the prevalence had tapered in few recent years[.2,3,4,5]

\section{Factor affecting myopia progression}

Baseline age was the strongest independent factor for myopia progression suggesting that younger children, particularly those 6 to 7 years old with atleast $-1.25 \mathrm{D}$ of myopia, are at risk of faster progression than older children regardless of other baseline characteristics. The younger the age at baseline the higher the myopia progression as well as the amount of myopia at 3 years despite the general similarity of myopia across age groups. One hypothesis for the faster progression at young ages is that the myopia that is already present in children by 6 or 7 years of age may be a different type from the myopia that occurs at ages 8 years and older (eg, more likely to have a genetic basis, more rapidly progressing, more likely to be at increased risk of high myopia).

The absence of a significant association between sex and axial elongation may be seen because of fact that females eyes are shorter and steeper cornea than those of male .But in some follow up studies it was shown that myopia progression and axial length progression occur same in both the sexes. we have seen that sex do not have great effect on progression of myopia in childhood. Well the difference was seen only in beginning of myopia or in early onset of myopia. 
There is always a difference in progression of myopia in different ethinic group. An school based study program was managed by COMET group in china, Singapore, with follow up period of 2 year, determine average annual progression rate in their population of myopic children from $-0.45 \mathrm{D}$ to $-0.60 \mathrm{D}$ and with $-0.35 \mathrm{D}$ per year.

It is seen in many researches that heredity has an influence on refraction is obvious. It was assume that heredity effect on refraction is also based on many physiological phenomena both in direct and other substantial factors.If we say that myopia is heredity than it will be not correct because as we have seen in many researches that in many other countries, where both parents are myopic but still the baby is emmetropic, so it is wrong to say that myopia is strongly associated with genetic only, there are other factors which also which lead to its progression. Axial length, a major endophenotype for refractive error is highly heritable which is influenced on one or more long arm of chromosomes 5[6]

Observational studies have shown that myopia is common among school children and educated people, and less common among non literate populations. Myopia has also been shown to be rare among occupational groups such as farmers and fishermen.It is seen that children those who are very much engaged in video games, television, laptop users are more prone to myopia in comparison to children likening outdoors games. But there is no significant correlation seen in children who $\mathrm{r}$ consuming more chocolate and they are myopic. In some of the report it is mentioned that near work lead to myopia progression but certain studies also shows that myopia progress not only due to near work but also having association with intensity and duration of work.[7,8,9,10,11,12]

Accommodation convergence have been suggested as the reasons sons for the development of myopia. If accommodation played a significant role in myopic progression. However, when a person with myopia reads without spectacles small changes in reading distance cause a greater percentage of changes in the amplitude of accommodation. There are constant saccadic back-and-forth movements of the eye during reading. These eye movements clearly cause repeated pressure and stretch pulses on the eye during reading . where as Skilled readers move their eyes, on average, every quarter of a second when reading. Accommodation is effective by the age of about four month and remain same for most purpose until the onset of presbyopia at the age of about 40 years .Still most of accommodation model draw their inspiration from the work of Helmholtz and his broad -brush view of the nature of mechanism and interaction between ciliary muscle zonule and lens is generally accepted .Exact role played by several of the ocular structure involved continue to be a matter of debate[13].

\section{Pathological changes in myopia}

Continuous lighting has found to produce axial elongation and myopia in rats and mice[14]. And axial elongation and flattening of the cornea resultant hyperopia in chicks. studies indicate that children spending more time outdoors are less prone to develop myopia and that this phenomenon is associated with exposure to light of higher intensity outdoors rather than with spending less time in reading or maintaining higher levels of physical activity. In one of these studies axial growth rate measured with ultrasound A-scan was not found to be significantly different during summer vacation and school year periods, although vitreous chamber depth in one subgroup was found to increase faster during the school year. Measurement of corneal curvature change or quantitative assessment of the relationship between length of day and myopia progression .But In a recent study from Guangzhou in southern China, myopia progression of 85 myopic children 6 to 12 years of age was found to be $40 \%$ lower in summer months compared with the winter, and axial elongation was found to be less in summer.

Experimentally, there is evidence supporting a protective role of light in development of excessive eye elongation and myopia, and it is possible that this effect is mediated partially by dopamine D2 receptors. Dopamine is reduced in the retina of visually deprived animals, and development of myopia can be prevented in these animals by administering dopamine agonists. it is well established that adenosine plays an important role in the response of the retina and retinal pigment epithelium to light.

Ocular refraction depends primarily of axial length, corneal curvature and lens power. At birth eye is hyperopic during childhood,active regulator process termed emmetropization harmonizes the expanding eye length to match the power of the cornea and lens to result in emmetropia. Emmetropization failure result in refractive error.[15]

\section{High myopia and anisometropia}

The clinical guidelines for the increased risk of amblyopia in myopes are $3.00 \mathrm{D}$ of anisometropia using these guideline, we found that a patient with high myopia and anisometropia had $84 \%$ chance of having amblyopia. Between 3.00 and $9.00 \mathrm{D}$ had a $79 \%$ to $81 \%$ chance of amblyopia. At $9.00 \mathrm{D}$ of anisometropia, amblyopia was present $100 \%$ of the time. This suggested the existence of an absolute amount of anisometropia above which amblyopia can be predicted.[16] 


\section{High myopia and astigmatism}

It is seen that astigmatism was a main contributing factor to amblyopia in patients with high myopia. But astigmatism can be an amblyogenic factor was not a major contributor to amblyopia. This suggested that the major contributing factor to amblyopia was the high myopia rather than astigmatism.

\section{High myopia and prematurity or low birth weight}

The positive correlation prematurity ,LBW, advanced stages of ROP, need for surgical intervention, and the severity of myopia and strabismus are well established[17] The term myopia of prematurity (MOP) was used to distinguish myopic children who were born prematurely with little or no ROP. It was generally believed though not universally held that premature infants had a greater risk of myopia than did their full-term. But in some of the papers it is accepted that low birth weight tented to increase of myopic severity as well as prevalence of anisometropia.

\section{Intervention for myopia progress}

The rate of progression of myopia is highest in young children, and the average age of stabilization of myopia is approximately 16 years. The onset myopia may occur at a relatively young age, leading to higher risks of high myopia (myopia at least_6.0 diopters [D]) in adulthood. Various studies have suggested several interventions that may retard the progression of myopia in children, which will lead to decreasing the severity of myopia at maturity. Contact lenses may flatten the cornea or may retard axial elongation. Muscarinic antagonist, atropine may act through muscarinic receptors to paralyze accommodation; or it may have a direct effect on scleral growth. In some of the cases it is seen that those eyes who where treated with atropine had more higher rate of myopia progression compared with eyes treated with placebo [18]. But absolute myopia progression after 3 years was significantly lower in the atropine group compare with placebo. As atropine is very effective in treatment of myopia because it act on one or more muscarinic receptor to differentially regulate expression level of specific receptor [19]. Adrenergic or blocking drugs may reduce raised intraocular pressure in high myopia. Bifocal lenses may reduce defective accommodative effort and improve retinal image quality in patients with high accommodative lag, thereby preventing potential aberrant eye growth[20]. We do not recommend soft contact lenses for the retardation of the progression of myopia, because the evidence suggests that there is no significant retardation of the progression of myopia. Soft contact lenses are associated with a higher risk of ocular complications compared with rigid gas-permeable lenses. Soft contact lens wear is not without attendant risks such as ocular infections; the maintenance and care of contact lenses may be an inconvenience mainly in children. The beneficial effects of atropine eye drops have also been shown in retrospective studies, non controlled trials, and nonrandomized controlled studies. The efficacy of atropine is supported by animal experiments, demonstrating that muscarinic acetylcholine receptors may affect eye and scleral growth.

Atropine eye drops may not be recommended for all myopic children, because the possible long-term side effects such as cataract formation and retinal toxicity, are largely unknown. Few studies have evaluated the possible adverse effects and complications of the use of anti cholinergic agents in growing eyes, especially over long periods of time. There are some combined therapy in which auricular acupoint where enhanced to lower level atropine eye drop. when used for myopia control in children the efficacy of this therapy was satisfactory but still axial length of eye should be further investigated [21].

\section{Conclusion-}

As this study belongs to myopia progression in children, though a lots of basic factors are responsible for its progression but still it is very Important to know that which factor playing more significant role for myopia progression in children .Use of atropine is also contradictory in long term use, so it is important to innovate some new drug or modality which stop myopia progression safely in shorter period of time.

\section{Reference}

[1]. Tong L, Huang XL, Koh AL, Zhang X, Tan DT, and Chua WH. Atropine for the treatment of childhood myopia: effect on myopia progression after cessation of atropine. Ophthalmology. 2009;116:572-579. [PubMed: 19167081]

[2]. Beuerman RW, Saw S-M, Tan DTH, Wong T-Y: Myopia: Animal Models to Clinical Trials. Singapore: World Scientific Publishing Co Pte Ltd 2010:345-346. Interventions for Myopia

[3]. B., Young, T. L., Mitchell, P., Varma, R. et al. (2010). Prevalence of refractive error inSingaporean Chinese children: the strabismus, amblyopia, and refractive error inyoung Singaporean Children (STARS) study. Invest. Ophthalmol. Vis. Sci. 51, 13481355

[4]. Ganesan, P. and Wildsoet, C. F. (2010). Pharmaceutical intervention for myopiacontrol. Expert Rev. Ophthalmol. 5, 759-787..

[5]. Rose KA, Morgan IG, Smith W, et al. Myopia, lifestyle, andschooling in students of Chinese ethnicity in Singapore and

[6]. Sydney. Arch Ophthalmol. 2008;126:527-530

[7]. Zhu G, Hewitt AW, Ruddle JB, et al. Genetic dissection of myopia: evidence for linkage of ocular axial length to chromosome 5q. Ophthalmology. 2008; 115:1053-1057. [PubMed: 17964656] 
[8]. Ip JM, Saw SM, Rose KA, et al . InvestOphthalmol Vis Sci. 2008; 49:2903-2910

[9]. Lu B, Congdon N, Liu X, et al. Associations between near work, outdoor activity, and myopia among adolescent studentsin rural China: the Xichang Pediatric Refractive Error Studyreport no. 2. Arch Ophthalmol. 2009;127:769-775.

[10]. Jones-Jordan LA, Mitchell GL, Cotter SA, et al. Visual activityPrior to and following the onset of juvenile myopia. InvestOphthalmol Vis Sci. 2011;52:1841-1850

[11]. Donovan L, Sankaridurg P, Ho A, et al. Myopia progression inChinese children is slower in summer than in winter. OptomVis Sci. 2012;89:1196-1202

[12]. Smith EL III, Hung LF, Huang J. Protective effects of highambient lighting on the development of form-deprivationmyopia in rhesus monkeys. Invest Ophthalmol Vis Sci. 2012; 53:421-428

[13]. Weizhong L, Zhikuan Y, Wen L, Xiang C, Jian G. A longitudinal study on the relationshipbetween myopia development and near accommodation lags in myopic children. OphthalmicPhysiol Opt. 2008; 28:57-61. [PubMed: 18201336]

[14]. Charman, W. Neil. "The eye in focus: accommodation and presbyopia." Clinical and experimental optometry 91.3 (2008): $207-225$.

[15]. Zhou X, An J, Wu X, et al. Relative axial myopia induced by prolonged light exposure in C57BL/6 mice. Photo chem Photo biol 2010;86:131-7

[16]. Stone RA, Khurana TS. Gene profiling in experimental modelsof eye growth: clues to myopia pathogenesis. Vision Res.2010;50:2322-2333

[17]. Pang, Yi, et al. "A prospective study of macular thickness in amblyopic children with unilateral high myopia." Investigative ophthalmology \& visual science 52.5 (2011): 2444-2449.

[18]. Quinn, Graham E., et al. "Progression of myopia and high myopia in the early treatment for retinopathy of prematurity study: findings to 3 years of age."Ophthalmology 115.6 (2008): 1058-1064.

[19]. 18-22 Tong L, Huang XL, Koh AL, Zhang X, Tan DT, Chua WH. Atropine for the treatment ofchildhood myopia: effect on myopia progression after cessation of atropine. Ophthalmology. 2009;116:572-579. [PubMed: 19167081]

[20]. Barathi, V. A. and Beuerman, R. W. (2011). Molecular mechanisms of muscarinicreceptors in mouse scleral fibroblasts: Prior to and after induction of experimentalmyopia with atropine treatment. Mol. Vis. 17, 680-6

[21]. Anstice, Nicola S., and John R. Phillips. "Effect of dual-focus soft contact lens wear on axial myopia progression in children." Ophthalmology 118.6 (2011): 1152-1161.

[22]. Hornbeak D, Young TL. Myopia genetics: A review of current research and emerging trends. Curr Opin Ophthalmol. 2009; 20:356362. [PubMed: 19587595] 